\title{
Le maintien des emplois pendant la période de l'état d'urgence à la suite de la Covid-19 : analyse critique des mesures instituées en RDC au regard du droit du travail
}

\author{
Par Juslain NSAMBANA BONKAKO KASILAM*
}

\section{Résumé}

Le devoir de sauvegarder les emplois a poussé les pouvoirs publics à prendre certaines mesures consistant notamment à interdire les licenciements massifs pendant la période de l'état d'urgence proclamé à la suite de la covid-19 en RDC. Par contre, il est de droit pour les entreprises confrontées aux difficultés économiques de procéder à leur réorganisation notamment par la réduction des effectifs de leurs travailleurs. Cet article examine l'applicabilité de cette interdiction au regard de la situation catastrophique des employeurs, en précisant à la fois la nature juridique de covid-19 en droit du travail et ses effets dans les relations professionnelles entre travailleur et employeur. Enfin, quelles perspectives pour l'Etat face à un phénomène de la nature de covid-19, en vue d'une efficacité dans la protection des emplois et la contagion de ses effets.

\begin{abstract}
The duty to safeguard jobs led the government to take certain measures, including prohibiting mass layoffs during the period of the state of emergency proclaimed following the covid-19 in the DRC. On the other hand, it is the right of companies facing economic difficulties to reorganize, in particular by reducing the number of their workers. This article examines the applicability of this prohibition in the light of the catastrophic situation of employers, by specifying both the legal nature of covid-19 in labour law and its effects in industrial relations between worker and employer. Finally, what are the prospects for the State in the face of a phenomenon of the nature of covid-19, with a view to its effectiveness in protecting jobs and the contagion of its effects?
\end{abstract}

\section{Introduction}

La pandémie de Covid-19 a, à travers le monde, entrainé des conséquences négatives dans plusieurs domaines de la vie. Il va de soi que le domaine de la santé mérite la primeur des

* Assistant du Professeur Jean-Michel KUMBU de la Faculté de droit de l'Université de Kinshasa; Apprenant au III ${ }^{\text {ème }}$ Cycle - Unikin, Chercheur au CREEDA (RDC); jnsambana@gmail.com. 
réflexions ${ }^{1}$ face à ce fléau mondialement désastreux ${ }^{2}$. Néanmoins, il ne fait d'ombre de doute qu'il n'a pas épargné la vie économique et, particulièrement, les entreprises qui se sont vues être obligées d'examiner diverses options juridiques existantes en vue de faire face auxdites conséquences, spécialement dans le domaine de la gestion du personnel ${ }^{3}$.

En République démocratique du Congo, comme dans d'autres pays menacés par ce monstre sanitaire, les pouvoirs publics ont été amenés à, non seulement, proclamer l'état d'urgence consistant à limiter certaines libertés individuelles pendant une certaine période ${ }^{4}$, mais également à prendre certaines mesures restrictives pendant la même période ${ }^{5}$. Parmi ces mesures, il y'a lieu de relever, en RDC, celle consistant au maintien des emplois qui implique l'interdiction pour les entreprises de procéder à des résiliations des contrats de travail pendant la période définie.

La mesure de maintien des emplois dans les entreprises a, certes, ces mérites en ce qu'elle a permis d'éviter l'hémorragie imaginable des résiliations des contrats de travail à la suite de la pandémie de Covid-19; par contre, elle n'a pas tenu compte de la charge sociale que subiraient les entreprises dont les activités ont été ralenties ou suspendues, avec comme conséquence que la productivité et les ressources générées ne peuvent plus supporter notamment les différentes charges sociales.

La gestion du personnel est, comme toutes les autres, une branche de l'entreprise qui dépend de la situation économique ou, mieux, de la productivité de celle-ci. C'est cette évidence qui justifie dans la plupart des législations, si pas toutes, qu'il soit prévu, en sus des

1 C'est notamment le cas du débat qui a envahi le monde autour de la mise en place d'un vaccin comme mesure de prévention; également plusieurs études ont été menées pour identifier les causes des écarts de contaminations entre, particulièrement les pays du continent africain et d'autres.

2 Les statistiques publiées font état de 68.252 .608 contaminations à travers le monde dont, 1.557 .343 personnes ont perdu la vie (statistiques rendues disponibles sur Wilkipédia, consulté en date du 11 décembre 2020).

3 L'on peut relever notamment diverses rencontres organisées dans le cadre de la Fédération des entreprises du Congo (FEC) en vue d'entrevoir des solutions adéquates en vue de sauver le peu d'entreprises existantes dans un pays en quête d'investisseurs.

4 L'article 85 de la constitution de la RDC du 18 février 2006, telle que modifiée par la loi n ${ }^{\circ} 11 / 002$ du 20 janvier 2011 portant révision de certains articles de la constitution du 18 février 2006. C'est en exécution de ces prérogatives constitutionnelles que certaines libertés individuelles ont été limitées; c'est le cas de la liberté de manifestation, de la liberté de religion par l'interdiction de cultes collectifs, de la liberté de mouvement par la fermeture des frontières et isolement de la Ville de Kinshasa, etc.

5 Conformément à l'article 145 de la constitution, le Président de la RDC a pris l'ordonnance ${ }^{\circ}$ 20/014 du 24 mars 2020 portant proclamation de l'état d'urgence sanitaire pour faire face à l'épidémie de covid-19 qui a mis en place certaines mesures, à savoir : des mesures sécuritaires sur le territoire national, des mesures relatives à l'exercice de la liberté, de l'organisation et du fonctionnement de la riposte, des mesures sanitaires et des mesures économiques. 
motifs liés à la personne du travailleur (la conduite et l'aptitude) ${ }^{6}$, la possibilité pour l'employeur de suspendre ou, dans l'extrême, de résilier le ou les contrats de travail pour d'autres motifs (économiques ou de force majeure).

Face à cette évidente possibilité légalement consacrée et au regard des motifs irréfutables justifiés par l'existence de Covid-19, les mesures ainsi imposées aux entreprises consistant au maintien des emplois pouvaient-elles être applicables? En d'autres termes, les employeurs étaient-ils tenus au respect scrupuleux de cette mesure face aux impératifs économiques de leurs activités?

Cette question principale nous pousse à nous interroger sur les effets de la pandémie à covid-19 sur les entreprises en RDC et ce que prévoit le droit congolais sur le comportement à adopter par les employeurs face à la pandémie de covid-19? Dans le souci légitime exprimé de protéger les emplois face à la menace et, à une certaine mesure, la victoire de la pandémie de covid-19 sur l'économie, quelle a été la réaction de l'Etat, en marge de la mesure de maintien des emplois, à l'égard des entreprises et quelles sont les perspectives?

La résolution de ce questionnement nous oblige de ressortir, d'abord, les effets économiques de la pandémie de Covid-19, particulièrement dans les pays à forte dépendance extérieure, ensuite, d'examiner les différentes possibilités légales des entreprises dans le domaine de gestion du personnel au passage de la pandémie de Covid-19 et, enfin, ressortir le rôle de l'Etat face aux réalités socio-économiques d'un tel fléau.

\section{A. Effets économiques de la pandémie de covid-19 en RDC : causes et perspectives}

Deux facteurs sont importants dans l'étude des conséquences économiques de la pandémie de covid-19, à savoir : le facteur de l'offre et celui de la demande, les deux formant ainsi l'essentiel des marchés économiques mettant en relation deux catégories d'acteurs indispensables de la vie des affaires, en l'occurrence : les producteurs (fournisseurs) et les consommateurs. A ces deux facteurs, s'ajoute la problématique de déplacement des produits du lieu de production à celui d'évacuation ou d'écoulement. Ces trois opérations sont indispensables dans la vie des acteurs économiques et nécessitent, pour leur réussite, un certain nombre des paramètres dont notamment : l'accès de la main d'œuvre à l'entreprise, le libre mouvement des personnes et des biens, le libre transfert entre opérateurs économiques (fournisseurs et commerçants grossistes et détaillants), etc.

La covid-19, une maladie planétaire, a imposé la prise de certaines mesures visant à freiner, sinon empêcher, sa propension et aggraver la crise à travers le monde. Parmi ces mesures, tenant compte du mode de transmission, la plupart des pays ont, entre autres, procédé par la fermeture des frontières, le confinement de certaines villes, la limitation des nombres des travailleurs appelés à prester sur un espace donné dans une entreprise, etc.

6 Article 62 de la loi $\mathrm{n}^{\circ} 015 / 2002$ du 16 octobre 2002 portant code du travail; Lire à ce sujet, $J M$. KUMBU ki NGIMBI, Droit social, Kinshasa, 2020; J. MASANGA PHOBA MVIOKI, Droit du travail, Kinshasa, 2017, p. 171; D. DIUMI SHUTSHA, Six leçons de droit du travail, Paris, 2018, p 583. 
Dans une étude publiée par l'Institut national de la statistique (INS), il est souligné que la crise de covid-19 menace de frapper de manière disproportionnée les pays et les mesures utilisées dans le processus d'endiguement de la transmission de la maladie sont susceptibles de créer des chocs économiques majeurs ${ }^{7}$. A l'issu de cette étude, des résultats ci-après ont été présentés : près de deux tiers des entreprises ayant connu une cessation d'activités au cours du mois de mai 2020, l'ont été à cause des mesures gouvernementales de lutte contre la pandémie de la covid-19. De manière globale, près d'une unité économique sur cinq n'a pas exercé d'activité au cours du mois de mai. Moins de $6 \%$ des entreprises de trois grandes villes de la RDC (Kinshasa, Goma et Lubumbashi) ont mis en congé technique ${ }^{8}$ des employés depuis le mois d'avril'.

La crise économique de la RDC a plusieurs causes ${ }^{10}$; mais celles qui ont concouru à l'existence de covid-19 tiennent principalement à l'extraversion de l'économie congolaise $^{11}$, à l'abandon des secteurs d'agriculture, de pêche et d'élevage, ainsi qu'à la forte concentration de l'économie dans les grandes villes du pays, du reste sensiblement touchées par la pandémie.

Le monde des affaires est actuellement assis sur la réalité de globalisation que de protectionnisme. La Chine est, face à l'Afrique, devenue incontournable dans le domaine économique. En effet, la présence économique de la Chine en Afrique, surtout en RDC, n'est plus à rechercher; chaque famille africaine, voire chaque citoyen africain, ne peut vivre en se passant des produits fabriqués en Chine. Pourtant, comme pays d'origine de Covid-19, le ralentissement des activités suivi des mesures étatiques de fermeture des frontières ou d'interdiction de vols en provenance ou en destination de la Chine a évidemment eu des conséquences sur les économies des pays africains, la RDC particulièrement.

Cette triste réalité s'observe même au niveau interne. En effet, il a été présenté statistiquement que les grandes villes ont été les plus touchées par la covid-19; ce qui a justifié notamment l'isolement de Kinshasa du reste du pays par l'interdiction des voyages entre la

7 INS, Mesures de l'impact du covid-19 sur les Unités économiques, Bulletin $n^{\circ} 01$, Kinshasa, juillet 2020, p.1.

8 Il s'agit d'une technique, non réglementée en droit congolais, consistant pour un employeur en difficultés de renvoyer un certain nombre des travailleurs pendant une certaine période sans résilier leurs contrats de travail. C'est donc, de facto, une suspension collective des contrats de travail permettant à l'employeur de surmonter la crise économique auquel il fait face.

9 INS, note 7, p. 1.

10 Ces causes de crise en RDC sont multiples; l'on peut relever notamment celles liées à la gouvernance en général et d'autres qui qui se rapportent à l'absence d'infrastructures propices aux activités économiques.

11 Dans une étude sur les impacts sanitaires et socioéconomiques de la covid-19 en RDC réalisée par le Gouvernement congolais, il a été souligné que du fait de leur forte ouverture sur l'extérieur et de la forte dépendance de leurs économies par rapport aux marchés mondiaux (cours des matières premières), plusieurs pays africains sont exposés aux affres de la pandémie de covid-19 ( $R D C$, Gouvernement, Impacts sanitaires et socioéconomiques de la covid-19 en République démocratique du Congo. Analyse prospective et orientations de la riposte multisectorielle, Kinshasa, mai 2020, p. 39). 
capitale de la RDC et les autres provinces, malgré une atténuation sur l'entrée des denrées alimentaires. Cette situation a eu des conséquences socio-économiques considérables.

Il est vrai que dans le cadre de la globalisation ou de la mondialisation qui tend à rendre, par le biais de l'intégration économique, le monde en un village planétaire dans lequel la circulation des biens et services ne doit souffrir d'obstacles étatiques, il est tout aussi important pour tout Etat de miser sur la production locale plutôt que faire dépendre l'économie sur les importations. Il est dès lors intéressant d'identifier le domaine d'activé qui offre plus garanties tant au niveau de la production que de la sécurité alimentaire et dont la mise en œuvre répond naturellement aux exigences de lutte contre les contaminations massives. En RDC, il s'agit évidemment de l'agriculture.

L'agriculture, longtemps laissée pour compte en RDC, a prouvé son importance dans le soutènement de l'économie et de la vie sociale pendant que le pays était sous embargo à l'arrivée du Président Laurent Désiré KABILA en 1997; ce dernier s'était appuyé sur l'agriculture pour assurer une certaine sécurité alimentaire au pays. Pendant une période de crise sanitaire, comme il en est le cas avec la pandémie de covid-19, la préoccupation majeure de la population est essentiellement tournée vers l'accès aux besoins élémentaires de la vie, à savoir notamment l'alimentation. L'agriculture est, contrairement au domaine minier qui est esclave de l'extérieur, un secteur d'activité qui peut à la fois faire développer un marché intérieur et faire générer beaucoup d'emplois en milieux ruraux, limitant ainsi l'exode de la population vers les grandes villes, exposées à des contaminations. Finalement, pour un pays à forte potentialité agricole, les effets économiques d'une pandémie comme la covid-19 pourraient être atténuées par la production agricole, laquelle résoudrait en même temps la question de la crise alimentaire pendant la même période. Au-delà de ces aspects, il y'a lieu de relever également le fait que la nature même de l'activité agricole expose moins les travailleurs à des risques de contamination due au rapprochement des personnes, la distanciation étant naturellement observée dans un tel domaine d'activité.

L'agriculture reste la perspective adéquate face à une telle réalité sanitaire aux effets multidimensionnels obligeant les uns et les autres à prendre certaines mesures pour y faire face. C'est le cas notamment des entreprises qui sont appelées à examiner les possibilités légales dans la gestion du personnel.

\section{B. Possibilités légales des entreprises dans le domaine de l'emploi face au passage de la pandémie à covid-19 : Suspension ou résiliation des contrats de travail}

Avant de présenter les différentes hypothèses consacrées à la survenance d'une telle situation dans le fonctionnement d'une entreprise, il nous semble nécessaire de rechercher la nature à accorder à la pandémie de Covid-19 dans les rapports contractuels entre employeur et travailleur. Ainsi, une question nous permet d'introduire cette réflexion, à savoir : si la Covid-19 peut être qualifiée de force majeure en droit. 


\section{La pandémie de Covid-19: un exemple de force majeure, une cause des difficultés économiques de l'employeur ou une simple maladie (non professionnelle) pouvant entrainer une incapacité de travail}

\section{La pandémie de covid-19 comme exemple de force majeure}

La force majeure est, au sens large, comprise comme tout événement imprévisible et insurmontable empêchant le débiteur d'exécuter son obligation ${ }^{12}$; elle est exonératoire. Dans tout contrat, sauf quelques spécificités légalement consacrées, les parties sont déliées de leurs obligations dès qu'il est démontré l'existence d'un événement imprévisible, insurmontable et non-imputable à qui que ce soit.

En matière du travail, il y'a force majeure lorsque l'événement survenu est imprévisible, inévitable, non imputable à l'une ou l'autre partie et constitue une impossibilité absolue d'exécution d'obligations contractuelles ${ }^{13}$. Ces caractéristiques permettent, le cas échéant, de se fixer sur la nature de force majeure de la pandémie de Covid-19. Dans cette démarche tendant à constater le cas de force majeure, l'on se demanderait si ces caractéristiques sont cumulatives ou alternatives pour parvenir à qualifier un événement. En effet, la conjonction " et », employée dans l'énumération des caractéristiques de force majeure en droit du travail, permet de justifier qu'il faut la réunion de tous ces éléments pour qualifier un événement de cas de force majeure en droit du travail. C'est dire que dans la démarche de l'inspecteur du travail tendant à constater le cas de force majeure ${ }^{14}$, son analyse consistera à parcourir toutes ces caractéristiques. L'absence d'une caractéristique fait perdre le régime de force majeure à un événement.

Revenant au cas particulier de la pandémie de Covid-19, il est facile à déceler certaines caractéristiques de force majeure, telles que l'imprévisibilité et la non-imputabilité; Cependant, le caractère inévitable et l'impossibilité absolue d'exécuter les obligations contractuelles peuvent être discutables, surtout dans des pays où la gestion de la pandémie semble avoir porté des fruits. Evoquer la pandémie de covid-19 comme cas de force majeure pour assoir la décision de l'employeur est discutable et peut faire naitre de litiges collectifs ou individuels du travail. Heureusement, encore faut-il qu'il soit honnête et formel, l'inspecteur du travail constitue une barrière importante à cet éventuel arbitraire de l'employeur.

Par contre, les conséquences de la Covid-19 au plan économique peuvent entamer gravement la santé financière de l'employeur et justifier à ce titre que soit retenu, non pas la pandémie elle-même, prise en tant que cas de force majeure, comme motif qui fonde la décision de l'employeur, mais plutôt des difficultés économiques résultant de l'inactivité de

12 Lexique des termes juridiques, $15^{\text {ème }}$ éd, Paris, Dalloz, 2005, p. 299.

13 Article 57, alinéa 2 du code du travail; lire également J. MASANGA PHOBA MVIOKI, note 6, p. 149.

14 Le législateur du code du travail a, pour éviter les abus et l'arbitraire des employeurs dans la qualification des cas de force majeure pour trouver en cela un motif en vue d'assoir leur décision, établi que le cas de force majeure est constaté par l'inspecteur du travail (article 57, alinéa 3 du code du travail). 
l'entreprise à la suite de la covid-19. D'ailleurs en RDC, il eut fallu que la mesure de confinement soit prise par les pouvoirs publics pour être retenue comme cause de suspension des contrats de travail de certains travailleurs pendant la période de l'état d'urgence. La force majeure pendant cette période ne reposait pas sur l'existence de la covid-19, mais plutôt sur des mesures prises par les pouvoirs publics qui peuvent être qualifiées de fait du prince $e^{15}$.

2. La pandémie de covid-19 comme cause des difficultés économiques de l'employeur

La pandémie, comme tout autre risque de la vie humaine, peut entrainer des conséquences tant dans le chef du travailleur que dans le fonctionnement de l'entreprise. Pour le travailleur, elle peut le soumettre à une impossibilité d'exécuter ses prestations; alors que pour l'employeur, elle peut entrainer des difficultés économiques. Comme dit supra, la pandémie de covid-19, en tant que maladie, ne peut, à notre point de vue, être retenue comme motif de suspension ou de résiliation du contrat de travail; sa qualification de cas de force majeure en droit du travail nous semble également moins pertinente. Par contre, ses conséquences sur le travailleur ou sur l'employeur peuvent s'avérer être des motifs de suspension ou de résiliation du contrat. De ce qui précède, l'on se situerait, soit, dans le champ de l'article 57 , point $1^{16}$, soit, de celui des articles 60 , a) et 62 du code du travail ${ }^{17}$ concernant respectivement la suspension du contrat de travail et la résiliation, comme différentes hypothèses légales à examiner au regard de l'existence de la pandémie de covid-19.

\section{Suspension et résiliation des contrats de travail comme possibilités de l'employeur confronté à l'existence de Covid-19}

1. De la suspension du contrat de travail fondée sur la covid-19: droits et obligations des parties

Le professeur Dieudonné DIUMI souligne que l'exécution du contrat de travail sans interruption n'est pas possible. La bonne exécution du contrat de travail peut se trouver perturbée par certaines situations, parfois imprévisibles qui donnent un juste motif pour les parties de ne pas exécuter leurs obligations ${ }^{18}$. Il précise que la suspension du contrat de travail

15 Le fait du prince évoque l'idée d'un acte de puissance publique rendant impossible l'exécution du contrat de travail (J. MASANGA PHOBA MVIOKI, note 6, p. 162).

16 Ici, le législateur n'évoque pas la maladie ou l'accident comme cause de suspension; c'est plutôt l'incapacité de travail qui se trouve être la conséquence de telle maladie ou tel accident, qui constitue la cause de suspension. Ceci est logique en ce qu'un travailleur peut être malade sans que celle-ci n'entrave l'exécution de ses obligations; la maladie ne peut donc entrainer la suspension de son contrat, c'est l'incapacité que peut en résulter.

17 Ces deux dispositions consacrant la possibilité de résilier le contrat de travail ne retiennent pas non plus la maladie, mais plutôt les conséquences de la maladie surtout, comme pour le cas d'une pandémie, si elle est de nature à paralyser le fonctionnement de l'entreprise. 
vise des cas où, compte tenu de certains événements extrinsèques au contrat de travail, l'exécution des principales obligations de ce contrat est paralysée. Elle ne vaut pas rupture du contrat de travail ${ }^{19}$. On dirait simplement que le contrat dort, il n'est pas mort.

La covid-19, comme maladie, fonderait la suspension du contrat de travail si elle entraine, au regard de l'article 57 du code du travail, l'incapacité de travail. La notion d'incapacité mérite alors d'être abordée; il y'a lieu de s'interroger sur le sens que le législateur social donne à ce concept dans ce cas précis : s'agit-il de l'incapacité au sens d'affaiblissement (physique ou intellectuel) ${ }^{20}$ dont atteindrait un travailleur à la suite d'une maladie? ou de l'impossibilité de travailler soit, parce que l'accès au lieu de travail est rendu difficile par prévention de risque de contamination, soit du fait d'une décision des pouvoirs publics?

L'incapacité de travail telle que prévue ci-dessus n'est pas techniquement synonyme de l'impossibilité ou l'empêchement auquel se serait butté un travailleur pour se rendre au lieu du travail ${ }^{21}$. Il s'agit d'une question liée à l'état du travailleur dont les facultés physiques ou mentales ont été affectées à la suite d'une maladie ou d'un accident; c'est ainsi qu'il est prévu des degrés d'incapacité pour la prise en charge en sécurité sociale.

Toutefois, la science juridique étant dynamique parce que reposant sur les faits et phénomènes sociaux, susceptible de surgir au fil du temps, les nouvelles réalités de covid-19 offrent également au droit social une matière à réflexion, imposant ainsi sa relecture ou son interprétation par les juges. En effet, sans méconnaitre d'autres maladies dangereuses existantes, la covid-19 est une maladie exceptionnelle tant dans ses modes de contamination que dans les effets qu'elle entraine, outre l'impuissance constatée des mécanismes sanitaires pour son éradication. Face à cette réalité, l'attention est prioritairement orientée vers les mécanismes de prévention en vertu desquels des mesures ont été prises tendant notamment à l'isolement de certains milieux mettant ainsi les travailleurs devant une impossibilité (non imputable à qui que ce soit) de fournir leurs prestations dans le cadre des contrats qui les lient à leurs employeurs.

La covid-19, à l'hypothèse où elle serait prise comme cas de force majeure, peut valablement entrainer la suspension du contrat de travail. Dans ce contexte, il est intéressant d'analyser les droits et obligations des parties pendant cette période de suspension du contrat.

19 Idem.

20 L'incapacité dans cette première interprétation est surtout soutenable en droit de la sécurité sociale, quand il s'agit de procéder à l'évaluation avant la prise en charge d'un assuré au titre de la branche des risques professionnels ou de la pension d'invalidité (articles 89 de la loi n ${ }^{\circ} 16 / 009$ fixant les règles relatives au régime général de la sécurité sociale et 76 de l'arrêté ministériel $n^{\circ} 146 \mathrm{du} 10$ novembre 2018 fixant les modalités d'affiliation des employeurs, d'immatriculation des travailleurs, de perception des cotisations, de liquidation et du service des prestations ainsi que les obligations qui incombent aux employeurs et aux travailleurs).

21 D'ailleurs, pour certaines catégories de travail dont les travailleurs peuvent, suivant les nouvelles technologies d'information et de la communication dans le cadre du télétravail, l'impossibilité d'atteindre le lieu du travail peut être couverte. En ce sens, l'incapacité ne peut s'analyser en impossibilité. 
En effet, comme droit particulier comportant des spécificités qui le démarquent parfois des principes de droit commun en matière des contrats, le droit du travail impose certaines exceptions aux règles ordinaires. C'est le cas du régime de maintien de certaines obligations dans le chef de l'employeur pendant la période de suspension du contrat alors que la prestation du travailleur n'est pas possible. Ce qui justifierait, en vertu du principe d'exceptio non adimpleti contractus ${ }^{22}$ (exception d'inexécution) cher dans tout contrat synallagmatique, que l'employeur soit dispensé de ses obligations principales. Les droits et obligations des parties pendant la suspension du contrat de travail pour cas de force majeure sont à examiner au regard de ce principe civiliste dans la mesure où, la force majeure n'entre pas dans le champ de l'article 59 du code du travail qui énumère des cas de suspension du contrat de travail pour lesquels certaines obligations sont maintenues.

En effet, à considérer la covid-19 comme cas de force majeure, la suspension du contrat de travail qu'elle entrainerait n'entre pas dans l'un des cas énumérés; ce qui confirme le principe civiliste ci-haut évoqué, dispensant ainsi l'employeur de l'obligation de rémunérer le travailleur dont le contrat est suspendu et ce, pendant toute sa durée.

Par contre, la covid-19 en tant que maladie et à condition d'entrainer une incapacité de travail, la suspension du contrat qui en découlerait ne dispense pas l'employeur de l'obligation de rémunérer le travailleur. En effet, pendant toute la durée de la suspension du contrat, l'employeur est soumis à l'obligation d'accorder deux tiers (2/3) de la rémunération en espèces et la totalité des allocations familiales au travailleur. Les avantages contractuels en nature $^{23}$ et le droit au logement sont également dus au travailleur ${ }^{24}$.

Outre ces obligations qui découlent de texte légal, d'autres résultent des dispositions conventionnelles et/ou contractuelles. Dans certaines entreprises, en effet, la convention collective prévoit notamment l'obligation d'assurer les soins médicaux aux travailleurs et à leurs familles jusqu'à un certain degré bien défini. En pareil cas, qu'il y'ait suspension à la suite de covid-19 au sens de l'article 57,1) du code du travail ou par simple contamination d'un travailleur imposant, en vue de prévenir d'autres contaminations, son isolement du milieu professionnel (hypothèse non-consacrée), l'employeur est tenu au maintien des obligations contractuelles.

2. De la résiliation du contrat pour cause de covid-19 : choix entre force majeure, nécessité de fonctionnement de l'entreprise ou motif économique et maladie susceptible d'entrainer une incapacité de travail

La résiliation du contrat de travail à la suite de covid-19, qu'elle soit considérée comme cas de force majeure ou comme cause de difficultés économiques ou la nécessité de la réorgani-

22 P. VAN OMMESLAGHE, Traité de droit civil Belge, tome I, Bruxelles, 1913, p. 873.

23 L'alinéa 2 de l'article 105 du code du travail reprend également les avantages en nature alors que parlant de la rémunération à l'alinéa $1^{\text {er }}$, et conformément aux dispositions de l'article $7, \mathrm{~h} \mathrm{du}$ même code, la valeur des avantages en nature figure déjà dans la rémunération.

24 Article 105 du code du travail. 
sation de l'entreprise, ou enfin comme maladie susceptible d'entrainer une incapacité de travail, obéit à un certain nombre d'exigences légales. L'employeur est tenu à certains préalables liés, d'une part à l'intervention d'une autorité publique (l'inspecteur du travail ou le ministre ayant le travail dans ses attributions) et, d'autre part, à l'observance d'un certain délai avant la prise de la décision.

S'agissant de la force majeure, l'inspecteur du travail est incontournable. Déjà en amont, c'est à lui qu'il appartient de qualifier la force majeure avant que l'employeur soit amené à suspendre le contrat de travail suivant ce motif. Cette exigence légale constitue une véritable garantie des travailleurs devant les employeurs qui peuvent toujours se servir arbitrairement de tel événement qui, en réalité, ne réunit pas les caractéristiques de force majeure. Dans l'hypothèse où la covid-19 serait retenue par l'inspecteur du travail comme cas de force majeure, la partie intéressée peut résilier le contrat après deux mois de suspension.

La résiliation éventuelle de contrat de travail à la suite de pandémie de covid-19, prise comme cas de force majeure, obéit à deux préalables : d'abord la décision de l'inspecteur examinant et constatant si la pandémie est constitutive de cas de force majeure et, ensuite, attendre l'écoulement de deux mois de suspension avant de résilier le contrat.

Concernant le motif économique ou celui lié aux nécessités de fonctionnement de l'entreprise, ayant comme cause l'existence de la pandémie de covid-19, l'employeur est également soumis à une exigence : la demande d'autorisation de licenciement du ministre ayant le travail dans ses attributions. L'employeur dépend donc de la suite à obtenir du ministre avant de procéder au licenciement pour cause de covid-19, si elle est considérée comme illustration de motif économique ou de nécessité de fonctionnement de l'entreprise ${ }^{25}$.

La pandémie de covid-19 a inévitablement imposé un autre régime de travail dans la plupart d'entreprises, sinon toutes, au point d'entrainer sensiblement la diminution de leurs activités dont les conséquences économiques ne peuvent empêcher leur restructuration. En outre, les mesures de confinement ou de fermeture des frontières, prises à la suite de covid-19, ont placé les entreprises, surtout les sociétés de transport aérien, devant une évidente nécessité de se réorganiser notamment par des mécanismes de suspension des contrats (qualifiés sans fondement légal en droit congolais de congé technique) ou de diminution des effectifs par la résiliation des contrats de travail.

Disons qu'il est évident que la covid-19 peut occasionner la résiliation de contrat de travail. L'employeur doit, pour ce faire, se conformer aux exigences légales. S'il la considère comme cas de force majeure, la constatation de l'inspecteur du travail ${ }^{26}$ et l'observance de

25 A ce sujet, l'article 7 de l'arrêté ministériel n 12/CAB.MIN/TPS/116/2005 fixant les modalités de licenciement des travailleurs fournit un certain éclairage sur ce qu'il faille comprendre par motif économique pouvant occasionner la résiliation du contrat. Cette disposition réglementaire cite notamment la diminution de l'activité de l'entreprise et sa réorganisation intérieure comme illustration de motif économique, pouvant fonder toute décision de licenciement. (Lire également, J. MASANGA PHOBA MVIOKI, note 6, pp. 181 et 182).

26 Article 57, alinéa 3 du code du travail. 
deux mois de suspension des contrats ${ }^{27}$ constituent des préalables à toute décision de résiliation; par contre, s'il tient à la présenter comme cause de motif économique ou de nécessité de fonctionnement, il est également tenu au respect de la procédure en recourant, le cas échéant, à la demande d'autorisation auprès du ministre ayant le travail dans ses attributions après avoir requis l'avis de la délégation syndicale ${ }^{28}$. Enfin, à l'hypothèse de considérer le corona virus comme maladie (non-professionnelle ou non-d'origine professionnelle), encore qu'il ait entrainé l'incapacité de travail dans le chef du travailleur, l'employeur n'a la possibilité de résilier le contrat qu'après six mois interrompus d'une telle incapacité29.

Un tel risque au plan social impose l'analyse de la responsabilité de l'Etat dans la protection des personnes face aux conséquences socio-économiques de la pandémie de covid-19.

\section{Responsabilité de l'Etat face aux conséquences de la pandémie de covid-19 dans le domaine de l'emploi}

Les pouvoirs publics ont une importante responsabilité en matière d'emploi. Si la constitution de la RDC prévoit que le travail est un droit sacré pour chaque congolais ${ }^{30}$, il en découle qu'il revient à l'Etat d'assumer l'obligation inverse, consistant à permettre à chacun de jouir de ce droit constitutionnellement garanti. C'est en ce sens que le constituant de 2006 a prévu que l'Etat garantit le droit au travail, la protection contre le chômage et une rémunération équitable et satisfaisante assurant au travailleur ainsi qu'à sa famille une existence conforme à la dignité humaine, complétée par tous les autres moyens de protection sociale, notamment, la pension de retraite et la rente viagère ${ }^{31}$.

Outre la réglementation édictée pour encadrer ce droit au travail, à savoir tous les instruments juridiques réglementant le domaine du travail et de la sécurité sociale, l'Etat est appelé, en des circonstances exceptionnelles, telle l'existence de la pandémie de covid-19, à prendre certaines mesures tendant à renforcer sa protection, toutefois, en veillant également sur l'équilibre à garantir entre, le cas échéant, les travailleurs et les employeurs.

27 Article 60, c) du code du travail.

28 En conformité aux dispositions de l'arrêté ministériel n 12/CAB.MIN/TPS/116/2005 du 26 octobre 2005 fixant les modalités de licenciement des travailleurs et de l'arrêté ministériel $\mathrm{n}^{\circ} 12$ / CAB.MIN/TPS/038/08 du 08 août 2008.

29 Article 60, a) du code du travail.

30 Cette disposition constitutionnelle nous semble entretenir une discrimination liée à la nationalité; elle aurait consacré ce droit à toute personne peu importe sa nationalité comme en ce qui concerne le droit à l'initiative privée posé à l'article 35 de la même constitution, quitte à renvoyer l'application de tel droit aux lois et règlements en matière du travail.

31 Article 36 de la Constitution du 18 février 2006, précitée. 


\section{Lutte contre les licenciements massifs à la suite de la pandémie de covid-19}

En marge des mesures contenues dans l'ordonnance du 24 mars 2020, proclamant l'état d'urgence sanitaire en RDC, bien d'autres mesures ont été prises pour réguler certains secteurs exposés aux effets néfastes de la pandémie; c'est le cas du domaine de l'emploi.

Comme il a été souligné avant, la pandémie de covid-19 constitue un danger pour les emplois. La prudence collective à observer pour limiter sa propagation est à la base de sérieux problèmes dans les entreprises, dont certaines, le cas de celles évoluant dans le secteur de transports des personnes (aérien, maritime ou terrestre), ont connu une baisse importante de leur chiffre d'affaire, et d'autres ont été momentanément fermées, c'est le cas des petites entreprises de services d'hôtellerie, de cafés, de location des salles et espaces pour cérémonies, sans oublier les structures d'enseignement qui ont aussi été fermées.

La mesure consistant à interdire le licenciement massif nous impose une certaine critique au regard non seulement du droit du travail, mais également de la justice sociale vis-àvis des employeurs confrontés aux mesures édictées dans l'ordonnance précitée, consacrant ainsi la fermeture pour certains et la réduction des activités pour d'autres. Il est vrai que pendant la période d'état d'urgence, qu'il soit sanitaire, sécuritaire ou autre, les principes de la vie normale en société se trouvent être perturbés pour faire face au phénomène à combattre. Cependant, il est invraisemblable d'interdire les licenciements massifs dans une entreprise fermée par les mêmes pouvoirs publics.

Les mesures imposées à certaines entreprises, de même que l'impossibilité pour d'autres d'accéder au lieu du travail à la suite des mesures de confinement, ont constitué une illustration de cas de force majeure, techniquement qualifié de fait de prince. De ce fait, il importe de le rappeler, l'employeur peut résilier le contrat après deux mois de suspension. L'on se souviendra que l'état d'urgence sanitaire, et par conséquent les mesures accompagnatrices, a duré plus de trois mois. C'est dire que légalement, les employeurs confrontés à ces mesures étatiques étaient fondés à procéder aux licenciements des travailleurs pour faire face aux impératifs économiques de l'heure. Malheureusement ou heureusement, le formalisme imposé consistant à obtenir l'autorisation préalable constitue également une barrière au licenciement, de sorte que la mesure ainsi prise à l'occasion de la pandémie n'a fait que renforcer ce contrepoids à l'égard de l'employeur, parfois de bonne foi.

$\mathrm{Au}$ lieu d'entretenir une telle équation dans le domaine de l'emploi, d'autres mesures tendant à accompagner les entreprises pour préserver leur santé économique et sauvegarder les emplois méritent d'être examinées.

\section{Quid des autres mesures d'accompagnement des entreprises en RDC face aux effets de la pandémie de covid-19: cas des allégements fiscaux et subventions étatiques}

Outre les mesures prises dans l'ordonnance n²0/041 du 24 mars 2020 portant proclamation de l'état d'urgence sanitaire pour faire face à l'épidémie de covid-19, certaines autres mesures tendant à soutenir les entreprises et les ménages sont également possibles pour atté- 
nuer les effets de cette maladie : c'est le cas des allégements fiscaux accordés, de la gratuité de la consommation d'eau et d'électricité, de certains appuis financiers de l'Etat sous la forme des subventions en faveur de certaines entreprises sensiblement touchées par la présence de la maladie. Qu'en est-il de ces mesures en RDC pendant la période de la covid-19?

S'agissant des allégements fiscaux, en RDC, ces mesures ont essentiellement porté sur deux impôts, en l'occurrence : l'impôt professionnel sur la rémunération (IPR) des fonctionnaires et autres agents de l'Etat ainsi que la taxe sur la valeur ajoutée (TVA) sur les produits alimentaires.

Cette mesure fiscale en faveur, d'une part, des seuls fonctionnaires et autres agents de l'Etat et, d'autre part, au profit de tout acheteur des produits alimentaires n'a pas été ressentie par les entrepreneurs confrontés à une crise imprévisible. S'il est vrai que les mécanismes de recouvrement des impôts, taxes et autres redevances ont été assouplis pendant la période de l'état d'urgence décrété, les créances fiscales y afférentes n'ont pas été dégrevées. En outre, discriminatoire qu'elle était, cette mesure n'a concerné que les salariés de l'Etat. Cette discrimination, quoique non décriée, n'est pas de nature à favoriser une justice équilibrée entre membres d'une même société (Etat). La morosité de la vie occasionnée par la pandémie de covid-19 n'a pas établi de différence entre salariés de l'Etat et d'autres; l'Etat, dans sa mission d'assurer une justice sociale à ses membres, devait étendre cette faveur à tous les salariés, publics et privés, confrontés à la crise de covid-19, comme il en a fait pour l'accès gratuit à l'eau et l'électricité ainsi que l'exonération à la TVA sur les produits alimentaires, ayant profité à tout consommateur.

S'agissant des subventions étatiques à accorder aux entreprises confrontées aux conséquences économiques de la covid-19, tout dépend des capacités économiques de l'Etat. L'octroi des subventions aux entreprises pour soutenir leur économie a toujours une incidence positive sur les emplois; cependant pour des pays économiquement faibles il est difficile, voire impossible d'accorder des appuis aux entreprises sous forme des subventions.

\section{Conclusion}

La pandémie de covid-19 n'a pas épargné les entreprises. Les activités économiques de certaines d'entre elles ont connu un ralentissement compte tenu des mesures sanitaires mises en place pour lutter contre la propagation de cette maladie dans les milieux professionnels. Pour faire face à cette pandémie, par la limitation de la propagation, les pouvoirs publics ont été amenés à proclamer l'état d'urgence sanitaire conformément aux dispositions constitutionnelles, en prévoyant des mesures à observer, lesquelles ont davantage accentué la crise économique des entreprises.

La survie des emplois dans une entreprise est foncièrement tributaire de sa santé économique qui, elle, dépend de l'exercice sans faille des activités. Cela suppose pour les travailleurs d'être placés dans les conditions d'exécuter leurs obligations professionnelles. La pandémie de covid-19, comme maladie non professionnelle dont les modes de transmission imposent l'observance de certains principes, constitue un danger imminent pour tous. En 
marge de ces principes sanitaires et d'hygiène, qualifiés de gestes barrières et dans sa mission d'assurer la protection des personnes et la sauvegarde des emplois, certaines mesures consistant à éviter les licenciements massifs ont été prises par l'Etat, mettant ainsi les employeurs devant une équation.

Cette étude a consisté à analyser cette équation au niveau des employeurs et situer la responsabilité de l'Etat face à une telle réalité sanitaire, spécialement dans la vie économique des entreprises. En effet, la première préoccupation impose la qualification de la pandémie de covid-19 en droit du travail, où il a été question de préciser si la covid-19 peut être qualifiée de cas de force majeure, ou de maladie (non professionnelle susceptible d'entrainer une incapacité de travail) ou encore comme cause de difficultés économiques; tout ceci permettant, conformément aux dispositions du code du travail, à l'employeur de suspendre les contrats de travail ou de les résilier. Nous avons estimé que la covid-19 ne peut, en tant que telle, suffire pour aboutir à des licenciements. A considérer le cas de force majeure, ce qui peut être pris comme illustration, c'est la mesure prise pour lutter contre la covid-19, tel le confinement, qui constitue un cas de force majeure dit fait du prince et non la maladie elle-même. De même, en tant que maladie, il faudrait pour cela que la covid-19 ait entrainé l'incapacité de travail pour le travailleur; cette hypothèse nous a imposé la réflexion sur le sens à donner à l'incapacité de travail, si la notion renverrait même à l'impossibilité pour le travailleur d'atteindre son lieu de travail du fait des mesures à observer ou essentiellement à l'incapacité entendue comme diminution des facultés physique ou morale d'un travailleur, au sens du droit de la sécurité sociale. Cependant, la covid-19 peut sans nul doute entrainer des difficultés économiques imposant de ce fait la réorganisation de l'entreprise par la réduction des effectifs. Ceci semble à notre avis concilier la pandémie à une cause de licenciement, même massif, des travailleurs. Toutefois, la procédure dans chacune des hypothèses est réglementée.

Outre ces mesures de sauvegarde des emplois, entachées par ailleurs d'injustice à l'égard des employeurs, l'Etat a accordé certaines faveurs discriminatoires en matière fiscale au profit de ses seuls salariés en les exonérant de l'impôt sur la rémunération, sans étendre telle exonération à d'autres salariés, pourtant confrontés à la même crise. Au-delà de cela, les employeurs semblent n'avoir pas connu un appui étatique susceptible d'atténuer les effets de la pandémie et consolider la mesure de sauvegarde des emplois.

Il y'a lieu de relever en définitive, qu'une telle crise devait faire repenser les pouvoirs publics sur les initiatives publiques à mettre en place, notamment dans le domaine d'agriculture, pour non seulement sauver l'économie nationale aux conséquences d'une telle pandémie, mais également sauvegarder les emplois et garantir la sécurité alimentaire pendant la période d'occupation virale. 


\section{Références bibliographiques}

\section{Textes juridiques}

- Constitution de la RDC du 18 février 2006, telle que modifiée par la loi n¹1/002 du 20 janvier 2011 portant révision de certains articles de la constitution du 18 février 2006.

- Loi n ${ }^{\circ}$ 015/2002 du 16 octobre 2002 portant code du travail

- Loi n ${ }^{\circ} 16 / 009$ fixant les règles relatives au régime général de la sécurité sociale

- Ordonnance n ${ }^{\circ}$ 20/014 du 24 mars 2020 portant proclamation de l'état d'urgence sanitaire pour faire face à l'épidémie de covid-19

- Arrêté ministériel n ${ }^{\circ}$ 12/CAB.MIN/TPS/116/2005 fixant les modalités de licenciement des travailleurs

- Arrêté ministériel n 146 du 10 novembre 2018 fixant les modalités d'affiliation des employeurs, d'immatriculation des travailleurs, de perception des cotisations, de liquidation et du service des prestations ainsi que les obligations qui incombent aux employeurs et aux travailleurs

\section{Ouvrages}

- DIUMI SHUTSHA D., Six leçons de droit du travail, Paris, Editions espérance, 2018

- INS, Mesures de l'impact du covid-19 sur les Unités économiques, Bulletin nº 01, Kinshasa, juillet 2020

- KUMBU ki NGIMBI JM., Droit social, Kinshasa, Editions de l'Institut africain des Droits de l'Homme et de la Démocratie (I.A.D.H.D.), 2020;

- Lexique des termes juridiques, $15^{\text {ème }}$ éd, Paris, Dalloz, 2005

- MASANGA PHOBA MVIOKI J., Droit du travail, Kinshasa, 2017

- $\quad R D C$, Gouvernement, Impacts sanitaires et socioéconomiques de la covid-19 en République démocratique du Congo. Analyse prospective et orientations de la riposte multisectorielle, Kinshasa, mai 2020.

- VAN OMMESLAGHE, Traité de droit civil Belge, tome I, Bruxelles, 1913 